nicht nur die angestammte Unterstützerschaft zu mobilisieren, die sich im homogenen Parteinetzwerk bewegt, sondern Überzeugungsarbeit bei jenen zu leisten, die sich nicht in diesem Netzwerk befinden, aber für manche Themen der Partei zu gewinnen sind. Dafür eignen sich das Internet als Medium und seine Nutzer als Trägergruppe in besonderer Weise.

Parteien verzeichnen hier also einerseits einen direkten Kontrollverlust im Sinne von aktiver Steuerung. Andererseits besteht die Chance, viel zu gewinnen: Bürger, die aus eigener Initiative heraus für Politik beziehungsweise politische Inhalte werben und damit der weitverbreiteten These von der Politikverdrossenheit entgegenstehen. Diese Aufgabe erfordert allerdings ein noch besseres Verständnis der Logiken dieses Mediums. Denn noch ist offen, wie sich die Aktivität der Internet-Nutzer tatsächlich in den nächsten Jahren entwickeln wird. Erstens ist kaum prognostizierbar, ob sich die Zahl der aktiven „Sender“ von wahlkampfbezogenen Botschaften weiter erhöhen wird, oder ob es sich dabei auch mittelfristig um eine relativ kleine Gruppe handeln wird. Damit geht auch die Frage der Glaubwürdigkeit einher: Wird der Schwarm der Internet-Nutzer Informationen aus der eigenen „Community“ in ähnlich hohem Maße vertrauen wie neutral präsentierten Nachrichten? Zweitens ist unklar, ob auch im Bereich der sozialen Netzwerke eine Diversifizierung einsetzen wird, die möglicherweise wiederum zu homogeneren Netzwerken führen könnte. Sicher ist, dass das Internet ein Raum ist, der riesiges Potenzial bietet, den aber sowohl Wahlkämpfer als auch Wahlkampfforschung noch längst nicht erschlossen haben.

\title{
Haupt- und Nebenwahlkämpfe in Deutschland und Österreich
}

\author{
Jens Tenscher
}

Die vergangene Wahl zum Deutschen Bundestag bleibt in vielerlei Hinsicht als Solitär in Erinnerung: Niemals zuvor gab es zwischen zwei aufeinanderfolgenden Bundestagswahlen so starke Veränderungen in den Stimmenanteilen ${ }^{1}$, und niemals zuvor beteiligten sich so wenige Menschen an einer nationalen „Hauptwahl“ in Deutschland. Offenkundig war es den Parteien nicht gelungen, im Wahlkampf genügend Aufmerksamkeit auf die anstehenden Wahlen zu lenken und die Stimmberechtigten zu mobilisieren. ${ }^{2}$ Tatsächlich strahlte die Kampagne im Sommer 2009 eine eigentümlich unaufgeregte, ja sedative Wirkung aus: Es war ein „Valium-Wahlkampf“3, der auch nur sporadisch ein nennenswertes Medienecho

1 Vgl. Bernhard Weßels, Splitting sichert den Wechsel. Mit taktischem Wahlverhalten verhindert der Bürger Schwarz-Rot, in: WZB-Mitteilungen, 2009, H. 126, S. 33 - 37, S. 33.

2 Vgl. Mona Krewel / Rüdiger Schmitt-Beck / Ansgar Wolsing, Geringe Polarisierung, unklare Mehrheiten und starke Personalisierung. Parteien und Wähler im Wahlkampf, in: Hans Rattinger I Rüdiger Schmitt-Beck / Sigrid Roßteutscher / Bernhard Weßels (Hrsg.), Zwischen Langeweile und Extremen. Die Bundestagswahl 2009, Baden-Baden 2011, S. 33 - 57.

3 Helmut Rinsum / Stephanie Grill, Valium-Wahlkampf der Kanzlerin, in: Werben \& Verkaufen, 2009, H. 40, S. $18-19$. 
erzielte. Im Fernsehen wurde so wenig wie seit 1990 und in den Tageszeitungen so selten wie seit 1994 nicht mehr über die anstehende Bundestagswahl berichtet. ${ }^{4}$

Die Ursachen für die Zurückhaltung, mit der sich die Parteien und Massenmedien engagierten, scheinen auf der Hand zu liegen: Die Folgen der globalen Wirtschafts- und Finanzkrise zwangen die politischen Protagonisten in ungewöhnlich hohem Maße zur konstruktiven Zusammenarbeit und zum politischen Handeln selbst in Wahlkampfzeiten. ${ }^{5}$ Auch die Medien schenkten diesem Thema überdurchschnittliche Beachtung. Schließlich konnten die zur Großen Koalition vereinten Sozial- und Christdemokraten nicht die ihnen in Bundestagswahlkämpfen eigentlich angestammten Angriffs- und Verteidigungspositionen einnehmen. Vielmehr versuchten beide, sich im Rahmen von Verkaufsstrategien die Erfolge der zurückliegenden Wahlperiode auf die eigenen Fahnen zu schreiben. So war es weithin den kleinen Oppositionsparteien und den Christsozialen überlassen, Konflikte zu inszenieren, medienträchtige Konfrontationen zu suchen und zu polarisieren. In gewohntem Maße blieb diesen aber eine überdurchschnittliche Medienresonanz verwehrt. ${ }^{6}$

Über diese gängige Lesart des Bundestagswahlkampfes 2009 hinaus mag noch ein weiterer Grund für die geringe Resonanz, die dieser in der Öffentlichkeit auslöste, ausschlaggebend gewesen sein: eine eingeschränkte Professionalität im Kampagnenmanagement der Parteien. Diese Vermutung knüpft an die populäre Annahme an, dass mit der Intensität, Visibilität und „Lautstärke“, mit der sich Parteien in Wahlkämpfen engagieren, die Wahrscheinlichkeit, dass die Massenmedien über diese berichten und auch die Aufmerksamkeit, mit der die Wähler den Wahlkampf verfolgen, steigen - eine Annahme, die sich mit Blick auf Europawahlkämpfe immer wieder bestätigt hat. ${ }^{7}$

Allerdings unterscheiden sich Wahlen zum Europäischen Parlament (EP) von nationalen Hauptwahlen, zum Beispiel der Bundestagswahl, nicht zuletzt in der ihnen zugewiesenen Bedeutung. ${ }^{8}$ Dies schlägt sich in vielfältiger Weise nieder: Erstens schenken die Bürger in der Regel Hauptwahlen mehr Aufmerksamkeit und Interesse, sind stärker involviert als zu

4 Vgl. Jürgen Wilke / Melanie Leidecker, Ein Wahlkampf, der keiner war? Die Presseberichterstattung zur Bundestagswahl 2009 im Langzeitvergleich, in: Christina Holtz-Bacha (Hrsg.), Die Massenmedien im Wahlkampf. Das Wahljahr 2009, Wiesbaden 2010, S. 339 - 372, S. 342; Winfried Schulz / Reimar Zeh, Die Protagonisten in der Fernseharena. Merkel und Steinmeier in der Berichterstattung über den Wahlkampf 2009, in: ebenda, S. 313 - 338.

5 Vgl. Richard Hilmer, Bundestagswahl 2009. Ein Wechsel auf Raten, in: ZParl, 41. Jg. (2010), H. 1, S. $147-180$.

6 Vgl. Frank Brettschneider / Marko Bachl, Die Bundestagswahl 2009 und die Medien, in: Politische Studien, 2009, H. 428, S. 46 - 55.

7 Vgl. Mark Franklin / Cees van der Eijk / Erik Oppenhuis, The Institutional Context. Turnout, in: Cees van der Eijk / Mark Franklin (Hrsg.), Choosing Europe? The European Electorate and National Politics in the Face of Union, Ann Arbor 1996, S. 306 - 331; Federico Ferrara / J. Timo Weishaupt, Get Your Act Together. Party Performance in European Parliament Elections, in: European Union Politics, 46. Jg. (2004), H. 3, S. 283 - 306; Markus Steinbrecher / Sandra Huber, European Elections' Turnout from 1979 to 2004, in: Michaela Maier / Jens Tenscher (Hrsg.), Campaigning in Europe - Campaigning for Europe, Berlin / Münster 2006, S. 15 - 30.

8 Vgl. bereits Karlheinz Reif/ Hermann Schmitt, Nine Second-Order National Elections. A Conceptual Framework for the Analysis of European Election Results, in: European Journal of Political Research, 8. Jg. (1980), H. 1, S. 3 - 44, S. 9; Andreas M. Wüst / Dieter Roth, Parteien, Programme und Wahlverhalten, in: Jens Tenscher (Hrsg.), Wahl-Kampf um Europa. Analysen aus Anlass der Wahlen zum Europäischen Parlament 2004, Wiesbaden 2005, S. 56 - 85. 
supra- oder subnationalen Nebenwahlkampfzeiten. ${ }^{9}$ Zweitens berichten die Massenmedien häufiger und sichtbarer über nationale Hauptwahlen als über Nebenwahlen. ${ }^{10}$ Drittens kann angenommen werden, dass sich auch die Parteien in Hauptwahlkämpfen stärker engagieren, dass sie sich professioneller aufstellen als bei Nebenwahlkämpfen. ${ }^{11}$

Die empirische Unterfütterung dieser Annahmen ist jedoch vergleichsweise schwach. Zwar gibt es eine Vielzahl an Studien, die sich auf deskriptiven Pfaden dem Kampagnenmanagement bei nationalen Hauptwahlen ${ }^{12}$ oder Europawahlen ${ }^{13}$ zugewendet haben. Auch ist die Zahl der empirischen, ländervergleichenden Untersuchungen von Haupt- oder europäischen Nebenwahlkämpfen in jüngster Zeit gestiegen. ${ }^{14}$ Gleichwohl stützt sich die Vermutung, dass Parteien einen Unterschied zwischen Haupt- und Nebenwahlkämpfen machen, bislang entweder auf vereinzelte länderspezifische Analysen ${ }^{15}$ oder auf Kampag-

9 Vgl. Andreas M. Wüst / Markus Tausendpfund, 30 Jahre Europawahlen, in: APuZ, B 23/24 (2009), S. 3 - 9, S. 5 f.

10 Vgl. Claes H. de Vreese / Susan A. Banducci / Holli A. Semetko / Hajo A. Boomgaarden, The News Coverage of the 2004 European Parliamentary Election Campaign in 25 Countries, in: European Union Politics, 7. Jg. (2006), H. 4, S. 477 - 504; Claes H. de Vreese / Edmund Laufl Jochen Peter, The Media and European Parliament Elections. Second-Rate Coverage of a Second-Order Event?, in: Wouter van der Brug / Cees van der Eijk (Hrsg.), European Elections and Domestic Politics. Lessons from the Past and Scenarios for the Future, Notre Dame 2007, S. 116 - 130; Hajo G. Boomgarden / Claes H. de Vreese / Holli A. Semetko, „Hast' es nicht gesehen?!“ Haupt- und Nebenwahlkämpfe in deutschen Fernsehnachrichten, in: Jens Tenscher (Hrsg.), Superwahljahr 2009. Vergleichende Analysen aus Anlass der Wahlen zum Deutschen Bundestag und zum Europäischen Parlament, Wiesbaden 2011, S. 181 - 197.

11 Vgl. Jens Tenscher, Professionalisierung nach Wahl. Ein Vergleich der Parteienkampagnen im Rahmen der jüngsten Bundestags- und Europawahlkämpfe in Deutschland, in: Frank Brettschneider I Oskar Niedermayer / Bernhard Weßels (Hrsg.), Die Bundestagswahl 2005. Analysen des Wahlkampfes und der Wahlergebnisse, Wiesbaden 2007, S. 65 - 95.

12 Vgl. für Deutschland Jens Tenscher, Bundestagswahlkampf 2002. Zwischen strategischem Kalkül und der Inszenierung des Zufalls, in: Jürgen Falter / Oscar W. Gabriel / Bernhard Weßels (Hrsg.), Wahlen und Wähler. Analysen aus Anlass der Bundestagswahl 2002, Wiesbaden 2005, S. 102 133; Klaus Kamps, Zur Modernisierung und Professionalisierung des Kampagnenmanagements. Die Kampagnenorganisationen im Vergleich, in: Karl-Rudolf Korte (Hrsg.), Die Bundestagswahl 2009. Analysen der Wahl-, Parteien-, Kommunikations- und Regierungsforschung, Wiesbaden 2010, S. $187-221$.

13 Vgl. Stefan Reiser, Parteienkampagne und Medienberichterstattung im Europawahlkampf 1989. Eine Untersuchung zu Dependenz und Autonomieverlust im Verhältnis von Massenmedien und Politik, Konstanz 1994; Jürgen Wilke / Jens Tangemann, Wahlkampfkommunikation zur Europawahl 1999, in: Thomas Knieper / Marion G. Müller (Hrsg.), Visuelle Wahlkampfkommunikation, Köln 2004, S. 13 - 44; Jens Tenscher, Mit halber Kraft voraus! Parteienkampagnen im Europawahlkampf 2004, in: ders., a.a.O. (Fn. 8), S. $30-55$.

14 Vgl. David L. Swanson / Paolo Mancini (Hrsg.), Politics, Media, and Modern Democracy. An International Study of Innovations in Electoral Campaigning and Their Consequences, Westport 1996; Susan A. Banducci / Holli A. Semetko, Campaign Engagement in a Cross-national Comparative Perspective. The Importance of Context, Conference on Mass Communication and Civic Engagement at the Anneberg School for Communication, University of Pennsylvania, 27. August 2003; Federica Bicchi / Jean Blondel / Palle Svensson, The European Parliament Campaign, Working Paper 2003; Claes H. de Vreese, Second-Rate Election Campaigning? An Analysis of Campaign Styles in European Parliamentary Elections, in: Journal of Political Marketing, 8. Jg. (2009), H. 1, S. 7 - 19; Wojciech Gagatek (Hrsg.), The 2009 Elections to the European Parliament. Country Reports, Florence 2010.

$15 \mathrm{Vgl}$. Jens Tenscher, a.a.O. (Fn. 11). 
nenanalogien und indirekte Vergleiche von Wahlkämpfen erster und zweiter Ordnung. ${ }^{16}$ Was noch aussteht, ist ein systematischer, direkter und quantifizierender Vergleich der Kampagnen im Vorfeld von Haupt- und Nebenwahlen, der über das Beispiel Deutschlands hinaus gehen würde. Um diesen bemüht sich der vorliegende Beitrag, der die Wahlkampfbemühungen der Parteien in Deutschland und Österreich im Vorfeld der zurückliegenden nationalen Haupt- und der europäischen Nebenwahl untersucht. Die Auswahl der Länder beziehungsweise der Kampagnen folgt einem „most similar systems design“: Die Länder ähneln sich nicht nur in ihren politischen Systemen (parlamentarische Demokratien) und in ihren moderat polarisierten, symmetrischen Fünf-Parteiensystemen, sondern sie gelten auch beide als Prototypen des demokratisch-korporatistischen, mitteleuropäischen Medienmodells. ${ }^{17}$ Überdies regierten zum Zeitpunkt der jüngsten national ausgetragenen Wahlen, der österreichischen Nationalratswahl vom 28. September 2008, der EP-Wahl vom 7. Juni 2009 und der Bundestagswahl vom 27. September 2009, in beiden Ländern Große Koalitionen, also Bündnisse von Sozialdemokraten und Konservativen. ${ }^{18}$ Schließlich gaben in beiden Ländern bei der vergangenen Hauptwahl so wenige Wähler wie selten zuvor ihre Stimme ab ${ }^{19}$; bei der europäischen Nebenwahl 2009 wurde zudem in Deutschland und Österreich die jeweils zweitniedrigste Wahlbeteiligung erzielt (in Deutschland 43,3 Prozent, in Österreich 46,0 Prozent). Da auch der situative Kontext mit der Dominanz der globalen Wirtschafts- und Finanzkrise in beiden Ländern ähnlich war, lassen sich Unterschiede, Besonderheiten und Gemeinsamkeiten in den Parteienkampagnen vor allem auf parteienspezifische Einflüsse der Mesoebene zurückführen. Diese stehen im Mittelpunkt des vorliegenden Beitrags, der sich den Wahlkampagnen der Parteien unter dem Blickwinkel der Professionalität widmet.

\section{Professionalität und Professionalisierung von Wablkampagnen}

Die Feststellung, dass sich die Art und Weise, wie Parteien Wahlkämpfe planen, organisieren und durchführen, in den zurückliegenden dreißig bis vierzig Jahren enorm gewandelt hat, gehört mittlerweile zum Kernbestand der politischen Kommunikationsforschung. ${ }^{20}$

16 Vgl. Roland Cayrol, European Elections and the Pre-Electoral Period. Media Use and Campaign Evaluations, in: European Journal of Political Research, 19. Jg. (1991), H. 1, S. 17 - 29; Jacques Gerstlé / Holli A. Semetko / Klaus Schönbach / Marina Villa, The Faltering Europeanization of National Campaigns, in: Pascal Perrineau / Gérard Grunberg / Colette Ysmal (Hrsg.), Europe at the Polls. The European Election of 1999, New York 2002, S. 59 - 77; Jens Tenscher, a.a.O. (Fn. 13); Claes H. de Vreese, a.a.O. (Fn. 14).

17 Vgl. Daniel C. Hallin / Paolo Mancini, Comparing Media Systems. Three Models of Media and Politics, Cambridge 2004, S. 143 - 197.

18 Während die Große Koalition im Nachkriegsdeutschland eine Seltenheit darstellt (1966 bis 1969 und 2005 bis 2009), ist sie in Österreich der Normalfall (in 38 von 66 Nachkriegsjahren). Seit 2007 wird in Österreich die Regierung von Sozialdemokraten und Österreichischer Volkspartei (ÖVP) gestellt, mit der SPÖ als stärkster, den Bundeskanzler stellender Fraktion.

19 Die Wahlbeteiligung bei der letzten österreichischen Nationalratswahl fiel mit 78,8 Prozent nur unwesentlich höher aus als der absolute Tiefstwert (78,5 Prozent), der bei der Wahl 2006 erzielt wurde.

20 Vgl. Patrick Donges, Amerikanisierung, Professionalisierung, Modernisierung? Anmerkungen zu einigen amorphen Begriffen, in: Klaus Kamps (Hrsg.), Trans-Atlantik - Trans-Portabel? Die Ame- 
Dabei verdeckt der Blick auf einige besonders anschauliche Symptome jüngeren Datums, wie das Aufkommen von "Spin Doctors“ und anderen „Consultants“, die Einrichtung von „War Rooms“ oder das „Social Web Campaigning“, bisweilen, dass sich dahinter ein langfristiger Wandlungsprozess verbirgt, der im Moment lediglich eine besondere Beschleunigung zu erfahren scheint. ${ }^{21}$ Zurückzuführen sind entsprechende Veränderungen der politischen Kommunikationspraxis, wie oftmals angemerkt, insbesondere auf ein Zusammenspiel aus Veränderungen im soziostrukturellen, politisch-kulturellen und medialen Umfeld: Konfrontiert mit einer größer werdenden Gruppe an rationalen, volatilen und bindungsunwilligen Individuen, mit wachsender Komplexität im Prozess der Politikherstellung und einer neuen Unübersichtlichkeit im Bereich der Massen- und „neuen“ Medien reagieren die politischen Akteure in Form unterschiedlichster Anpassungsleistungen. ${ }^{22} \mathrm{Zu}$ nennen sind hier insbesondere:

(1) das verstärkte Bemühen der Parteien und politischen Eliten um eine konsequente Ausrichtung ihres Handelns auf die Wählerumwelt, den Wahlkampf und den Wahlerfolg (Elektoralisierung);

(2) die nachhaltige Orientierung von politischen Akteuren an journalistischen Erwartungen beziehungsweise medialen Logiken und Formaten (Medialisierung) ${ }^{23}$;

(3) das Bemühen um ein dauerhaft angelegtes, strategisch geplantes, rationales und effektives Kommunikationsmanagement (Professionalisierung).

Vor diesem Hintergrund lässt sich eine Fülle von nachhaltigen Transformationen der organisatorischen Strukturen und der alltäglichen politischen Kommunikationspraktiken von Parteien und anderen politischen Akteuren beobachten. ${ }^{24}$ Stark vereinfacht sind diese in den vergangenen Jahren immer wieder drei Phasen zugeordnet worden: vormodern, mo-

rikanisierungsthese in der politischen Kommunikation, Wiesbaden 2000, S. 27 - 40; Ralph Negrine, The Long Road to Professionalisation. Campaigning in Europe vs. Campaigning for Europe, in: Michaela Maier / Jens Tenscher, a.a.O. (Fn. 7), S. 31 - 44; Klaus Kamps, a.a.O. (Fn. 12); Christina Holtz-Bacha, Wahljahr 2009. Professionalisierung verzögert?, in: dies., a.a.O. (Fn. 4), S. $7-21$.

21 Vgl. Pippa Norris, A Virtuos Circle. Political Communications in Postindustrial Societies, Cambridge 2000; Stylianos Papathanassopoulos / Ralph Negrine / Paolo Mancini / Christina Holtz-Ba$c h a$, Political Communication in the Era of Professionalisation, in: dies. (Hrsg.), The Professionalization of Political Communication, Bristol / Chicago 2007, S. 9 - 25; Jesper Strömbäck, Selective Professionalisation of Political Campaigning. A Test of the Party-Centered Theory of Professionalised Campaigning in the Context of the 2006 Swedish Election, in: Political Studies, 57. Jg. (2009), H. 1, S. 95 - 116, S. 96 f.

22 Vgl. Winfried Schulz, Politische Kommunikation, Theoretische Ansätze und empirische Befunde, Wiesbaden 2008, S. 31 - 37.

23 Der Begriff der Medialisierung wird hier synonym mit dem Terminus „Mediatisierung“ und dem englischsprachigen Äquivalent „Mediatization“ genutzt. Gemeint ist damit die voranschreitende Orientierung und Anpassung politischer Akteure gegenüber den Strukturen und Handlungsweisen der Massenmedien, vgl. Patrick Donges, Medialisierung der Politik. Vorschlag einer Differenzierung, in: Patrick Rössler / Friedrich Krotz (Hrsg.), Mythen der Mediengesellschaft, Konstanz 2005, S. $321-339$.

24 Vgl. Klaus Kamps, Politisches Kommunikationsmanagement. Grundlagen und Professionalisierung moderner Politikvermittlung, Wiesbaden 2007; Uwe Jun, Parteien, Politik und Medien. Wandel der Politikvermittlung unter den Bedingungen der Mediendemokratie, in: Frank Marcinkowski / Barbara Pfetsch (Hrsg.), Politik in der Mediendemokratie, PVS-Sonderheft 42, Wiesbaden 2009, S. $270-295$. 
dern und postmodern. Diese Phasen würden sich durch je spezifische Kommunikationsmodi, -strukturen und -strategien auszeichnen. ${ }^{25}$ Bei genauerem Hinsehen erweist sich dieses oft bemühte „Phasenmodell“ jedoch als zu undifferenziert. Es vernachlässigt in hohem Maße länder-, parteien- und wahlspezifische Charakteristika. So ist es durchaus denkbar, dass in ein und demselben Land unterschiedliche Parteien im Rahmen von Hauptund Nebenwahlkämpfen gleichzeitig auf als „postmodern“, „modern“ und „vormodern“ klassifizierte Kommunikationsmodi zurückgreifen, dass sie auf manche Kommunikationsmittel ganz bewusst verzichten und andere stärken, um ihre je spezifischen Wahlkampfziele zu erreichen.

Tatsächlich scheint gerade dieser Mix an strategischen und strukturellen Kommunikationskomponenten das zentrale Kennzeichen professioneller Kampagnenführung zu sein. ${ }^{26}$ Der Begriff der Professionalisierung würde demnach nicht für eine bestimmte Phase stehen, sondern vielmehr auf einen Prozess verweisen: „a process of change [...] that [...] brings a better and more efficient organisation of resources and skills in order to achieve desired objectives, whatever they might be “27. Um im Zusammenhang mit Wahlkämpfen herauszufinden, wie professionell das Kampagnenmanagement einer Partei zu einem bestimmten Zeitpunkt ausfällt, bedarf es also des Blicks auf die Art und Weise des Einsatzes der zur Verfügung stehenden Mittel vor dem Hintergrund der zu erreichenden Möglichkeiten und Ziele einer Partei. Diese Ziele sind - wie die Mittel - keineswegs bei allen Parteien gleich, denn sie reichen von Stimmenmaximierung über Policy-Zentrierung bis hin zur Verhinderung von bestimmten Regierungsbündnissen. ${ }^{28}$

Eine derartige Annäherung an das Phänomen der Professionalität der Kampagnenkommunikation setzt einen Perspektivenwechsel voraus: weg von der Makroebene und hin zur Mesoebene der involvierten Parteien. Erst die komparative Analyse ermöglicht Aussagen über die Art und Weise der modernisierungsbedingten Anpassungsleistungen beziehungsweise die mit dem Einsatz bestimmter Mittel verbundenen Intentionen einer Partei. Durch entsprechende, quantifizierende Analysen kann dann das Ausmaß der Professionalität der Kampagnenkommunikation einer Partei in Relation zu anderen Parteien eruiert werden. ${ }^{29}$ Dabei muss die empirische Überprüfung dem Umstand Rechnung tragen, dass sich die Professionalität politischer Kommunikation (und nicht nur der Kampagnenkommunikation) sowohl in struktureller, organisatorischer Hinsicht, das heißt in Bezug auf die Ressour-

25 Vgl. Jay G. Blumler / Dennis Kavanagh, The Third Age of Political Communication. Influences and Features, in: Political Communication, 16. Jg. (1999), H. 3, S. 209 - 230; Pippa Norris, a.a.O. (Fn. 21), S. 137 - 145; Fritz Plasser / Gunda Plasser, Globalisierung der Wahlkämpfe. Praktiken der Campaign Professionals im weltweiten Vergleich, Wien 2002, S. 22 - 25.

26 Vgl. Jens Tenscher, a.a.O. (Fn. 11); Heiko Giebler / Andreas M. Wüst, Individuelle Wahlkämpfe bei der Europawahl 2009. Länderübergreifende und ebenenspezifische Befunde, in: Jens Tenscher, a.a.O. (Fn. 10), S. $121-152$.

27 Stylianos Papathanassopoulos / Ralph Negrine / Paolo Mancini / Christina Holtz-Bacha, a.a.O. (Fn. 21), S. 10, Hervorhebung des Autors.

28 Vgl. Rachel K. Gibson / Andrea Römmele, A Party-Centered Theory of Professionalized Campaigning, in: Harvard Journal of Press/Politics, 6. Jg. (2001), H. 4, S. 31 - 43, S. 26 f.

29 Vgl. Jesper Strömbäck, a.a.O. (Fn. 21); Rachel K. Gibson / Kim Jucknat / Andrea Römmele, Professionalisierte Kampagnenführung. Eine systematische Messung, in: Oscar W. Gabriel / Bernhard Weßels I Jürgen Falter (Hrsg.), Wahlen und Wähler. Analysen aus Anlass der Bundestagswahl 2005, Wiesbaden 2009, S. 457 - 483; Rachel K. Gibson / Andrea Römmele, Measuring the Professionalization of Political Campaigning, in: Party Politics, 15. Jg. (2009), H. 3, S. 321 - 339. 
cen und Kampagnenvoraussetzungen („Hardware“), als auch in strategischer Hinsicht, das heißt in Bezug auf die Kampagnenausführung („Software“), manifestieren kann - eine Differenz, die mitunter vernachlässigt wird, denn die meisten Kampagnenstudien „messen“ vor allem die strukturelle Seite des Wahlkampfs. ${ }^{30}$ Dem ist entgegenzuhalten, dass sich modernisierungsbedingte Veränderungen der politischen Kommunikation nicht nur in der Organisationsstruktur niederschlagen (das heißt in Bezug auf finanzielle, personelle, zeitliche, materielle, infrastrukturelle und kommunikationsbezogene Ressourcen), sondern immer auch in der Art und Weise der Wahlkampfführung, in der „Tonalität“ einer Kampagne, im Umgang mit den politischen Kontrahenten, im Kommunikationsmix, in Bezug auf Inhalte und Personen - kurz: in der strategischen Ausrichtung. ${ }^{31}$ Wenn es eine Möglichkeit gibt, beide Dimensionen - Kampagnenstrukturen und Kampagnenstrategien - zu messen, kann hieraus der „Professionalitätsgrad“ zu einem gegebenen Zeitpunkt erschlossen werden. Dieser kann wiederum in Beziehung zur Kampagnenprofessionalität der Parteien desselben oder eines anderen Landes gesetzt werden. Hieraus lassen sich Auskünfte über partei- und länderbezogene Charakteristika, aber auch über kontextunabhängige Merkmale der Wahlkampfführung ableiten.

Erst der longitudinale Vergleich zwischen mindestens zwei Messzeitpunkten ermöglicht darüber hinaus Aussagen über Veränderungen in der Professionalität des Kampagnenmanagements, also über den Prozess der Professionalisierung. ${ }^{32}$ Beide Perspektiven nimmt der vorliegende Beitrag ein, indem er die Parteienkampagnen anlässlich zweier a priori als unterschiedlich wichtig eingestufter Wahlen, der nationalen Haupt- und der europäischen Nebenwahl, in zwei Ländern untersucht. Hierdurch kann zum einen verdeutlicht werden, dass auch ein und dieselben Parteien einen kontextspezifisch divergierenden Einsatz ihrer Mittel favorisieren, also in recht unterschiedlichem Maße professionell agieren. Zum anderen lassen sich Unterschiede, Gemeinsamkeiten und länderbezogene Spezifika der Wahlkämpfe überprüfen.

\section{Methode}

Die empirische Messung der Professionalität von Kampagnen setzt das Vorhandensein entsprechender Indikatoren voraus. Allerdings gibt es noch kein vereinbartes Indikatorenset, auf das zurückgegriffen werden könnte. ${ }^{33}$ Vielmehr zeichnen sich zwei Operationalisie-

30 Vgl. Jesper Strömbäck, a.a.O. (Fn. 21); Rachel K. Gibson / Andrea Römmele, a.a.O. (Fn. 29); Christina Holtz-Bacha, a.a.O. (Fn. 20).

31 Vgl. Jens Tenscher, a.a.O. (Fn. 12); Christina Holtz-Bacha, Professionalisation of Politics in Germany. Campaigning in Europe vs. Campaigning for Europe, in: Stylianos Papathanassopoulos / Ralph Negrine / Paolo Mancini / Christina Holtz-Bacha, a.a.O. (Fn. 21), S. 63 - 79.

32 Vgl. Christina Holtz-Bacha, a.a.O. (Fn. 20), S. 15. Auf diese Differenzierung zwischen der Professionalität der politischen Kommunikationspraxis zu einem bestimmten Zeitpunkt einerseits und dem Prozess der Veränderungen zwischen mindestens zwei Messzeitpunkten, das heißt der Professionalisierung, andererseits, wird allzu oft verzichtet. Tatsächlich finden sich in einer ganzen Reihe an empirischen Studien zum Teil generalisierte Aussagen über Veränderungen, die auf Basis von Momentaufnahmen getroffen werden; vgl. Jesper Strömbäck, a.a.O. (Fn. 21); Claes H. de Vreese, a.a.O. (Fn. 14).

33 Vgl. Rachel K. Gibson / Kim Jucknat / Andrea Römmele, a.a.O. (Fn. 29), S. 459. 
rungsvarianten ab: (1) Der von Gibson und Römmele ${ }^{34}$ entworfene und von Strömbäck ${ }^{35}$ modifizierte CAMPROF-Index nimmt ausschließlich die organisatorische Kampagnenseite in den Blick und betont dabei den Gebrauch von Onlinemedien zur internen und externen Kommunikation. In die Kritik geraten ist dieser Index zum einen wegen seiner Fokussierung auf Parteien und Wahlkämpfe, zum anderen wegen seiner (Über-)Betonung der Onlinekommunikation, die einen longitudinalen Vergleich erschwert. ${ }^{36}$ (2) Eine Alternative, die nicht nur raum- und zeitungebunden ist, sondern sowohl die strukturelle als auch die strategische Seite politischer Kommunikation erfasst, ist der vom Autor vorgeschlagene Professionalisierungsindex. ${ }^{37}$ Dieser wurde anlässlich des Europawahlkampfes 2009 in Deutschland, Schweden, Finnland und Österreich getestet und entsprechend modifiziert. ${ }^{38}$ Als zentrale Komponenten zur Messung des Professionalitätsgrades einer Parteienkampagne (aber auch der Kommunikationsaktivitäten anderer politischer Akteure) haben sich dabei die folgenden Merkmale herauskristallisiert:

- Kampagnenstrukturen (acht Komponenten): Größe des Wahlkampfbudgets und des Mitarbeiterstabs, Ausmaß der Zentralisierung der Organisation und der Externalisierung von Kampagnenmaßnahmen, Ausdifferenzierung der internen Kommunikationsstrukturen, Art und Umfang der Rückkopplung, Ausmaß der Gegnerbeobachtung sowie Dauer der Kampagne.

- Kampagnenstrategien (sieben Komponenten): Ausmaß der Zielgruppenorientierung und der Narrowcasting-Aktivitäten ${ }^{39}$, Relevanz der kostenpflichtigen und -freien Medien sowie der so genannten Talkshowisierung, Ausmaß des Ereignis- und Newsmanagements sowie der Personalisierung.

Die Ausprägungen dieser Dimensionen werden entsprechend ihrer Intensität beziehungsweise des Ausmaßes des Auftretens anhand unterschiedlich stark differenzierter Skalen erhoben und zu entsprechenden Indizes gebündelt (vgl. Anhang). Die Zusammenfassung der Elemente zu Indizes ermöglicht es, das ganze Spektrum von vermeintlich "traditionellen“ (oder „vormodernen“), „modernen“ und „postmodernen“ Kampagnencharakteristika gleichsam zu zwei zentralen „Kampagnenbündeln“ - Strukturen und Strategien - zu verdichten. Da es bislang theoretisch wie empirisch unklar ist, ob und in welchem Maße die einzelnen Indikatoren dabei gewichtet werden müssen ${ }^{40}$, werden hier für die Berechnungen standardisierte Werte (z-values) genutzt. Auf dieser Basis wurden die beiden Indizes aus Gründen der Anschaulichkeit auf Werte zwischen 0 und 100 skaliert. Ein Wert von 80 würde entsprechend signalisieren, dass die Partei einen Professionalitätsgrad von 80 Prozent

34 Vgl. Rachel K. Gibson / Andrea Römmele, a.a.O. (Fn. 28 und 29).

35 Vgl. Jesper Strömbäck, a.a.O. (Fn. 21).

36 Vgl. Christina Holtz-Bacha, a.a.O. (Fn. 20), S. 13 f.

37 Vgl. Jens Tenscher, a.a.O. (Fn. 11).

38 Vgl. Jens Tenscher, Defizitär - und trotzdem professionell? Die Parteienkampagnen im Vergleich, in: ders., a.a.O. (Fn. 10), S. 65 - 95; ders. / Juri Mykkänen / Tom Moring, Modes of Professional Campaigning. A Four-Country-Comparison in the European Parliamentary Elections 2009, in: International Journal of Press/Politics, 17. Jg. (2012), H. 2, S. 145 - 168.

39 Gemeint sind damit zielgruppenorientierte, direktkommunikative Maßnahmen wie zum Beispiel Direct Mailings, Telefon-, E-Mail- oder SMS/MMS-Aktionen, aber auch Hausbesuche bei potentiellen Wählern.

40 Vgl. Rachel K. Gibson / Kim Jucknat / Andrea Römmele, a.a.O. (Fn. 29), S. 461. 
erreicht hätte. Die interne Konsistenz der beiden Indizes wurde überprüft. Sie ist mit .69 für den Index Kampagnenstrukturen und .72 für den Index Kampagnenstrategien zufriedenstellend (Cronbach's Alpha). Somit sind beide Indizes, wie auch andernorts festgestellt ${ }^{41}$, durch eine hohe interne Validität gekennzeichnet.

Neben der Frage der Validität der Indizes stellt sich die Frage nach der Verlässlichkeit der Messung der einzelnen Komponenten. Um diese zu gewährleisten, basieren die Indizes vorwiegend auf objektiv messbaren, das heißt vom Forscher unabhängig erfassbaren Kampagenkriterien. Diese werden ergänzt durch subjektive Einschätzungen der verantwortlichen Wahlkampfmanager. Deren Ansichten bieten, insbesondere wenn es um strategische Fragen geht, die bestmögliche Annäherung an die Kampagnenrealität, wie sie sich für die einzelnen Parteien und deren Mitarbeiter darstellt. ${ }^{42}$ In diesem Zusammenhang stützt sich die vorliegende Analyse einerseits auf schriftliche Befragungen der deutschen Wahlkampfverantwortlichen beziehungsweise auf teilstandardisierte mündliche Interviews mit den österreichischen Wahlkampfmanagern der in den jeweiligen Parlamenten vertretenen Parteien. Der Fragebogen umfasste 21 Fragen. Die Interviews dauerten im Durchschnitt 45 Minuten. Die Akteure (ein Verantwortlicher pro Partei) wurden im unmittelbaren Anschluss an die Nationalratswahl 2008, die Europawahl 2009 und die Bundestagswahl 2009 befragt. ${ }^{43}$ Die Reliabilität der subjektiven Einschätzungen der Wahlkampfverantwortlichen wurde durch Cross Checks innerhalb des Fragebogens überprüft. Sie ist zufriedenstellend, weswegen auf eine wiederholte Befragung derselben Personen verzichtet wurde. Untersuchungsleitend sind die folgenden Annahmen:

(1) Aus der „Nebenwahlthese“ (siehe oben) lässt sich ableiten, dass die Professionalität der Parteikampagnen im Vorfeld der EP-Wahl niedriger als zu Hauptwahlkampfzeiten ausfallen dürfte. Diese Annahme (H1) müsste auf alle Parteien, unabhängig von ihrer parlamentarischen Rolle oder der Länderzugehörigkeit, zutreffen.

41 Vgl. Jens Tenscher / Juri Mykkänen / Tom Moring, a.a.O. (Fn. 38); Jens Tenscher / Juri Mykkänen, Transformations in Second-Order Campaigning. A German-Finnish Comparison of Campaign Professionalism in the European Parliamentary Elections 2004 and 2009, in: Party Politics (in Begutachtung).

42 Vgl. auch Rachel K. Gibson / Kim Jucknat / Andrea Römmele, a.a.O. (Fn. 29), S. 462 - 263. Die Alternative bestünde darin, auf Einschätzungen externer Wahlkampfbeobachter (zum Beispiel Journalisten, „Experten“) zurückzugreifen oder diese zur Validierung zu nutzen. Deren Antworten mögen bisweilen „ehrlicher" als jene der direkt Betroffenen sein. Ob sie deswegen näher an der „Kampagnenrealität“ einer Partei sind, ist eine offene Frage. Es muss jedoch darauf hingewiesen werden, dass die im Anschluss an den Wahlkampf geäußerten Einschätzungen der Kampagnenverantwortlichen immer auch den Erfolg beziehungsweise Misserfolg der Parteien am Wahltag widerspiegeln. Einzelne Kampagnenmaßnahmen und allgemeine Beurteilungen des Wahlkampfs sind insofern durch das jeweilige Wahlergebnis „gefiltert", gegebenenfalls verzerrt und einzelne Antworten „rechtfertigen“ im Nachhinein bestimmte Maßnahmen. Dies gilt es bei der Interpretation der Auskünfte und Befunde insbesondere deswegen zu beachten, weil sich die Rücksendung der Fragebögen in Einzelfällen bis zu sechs Monate hinzog.

43 Während sich die FPÖ nicht an der Befragung zum Nationalratswahlkampf 2008 beteiligte, nahm das von Jörg Haider gegründete Bündnis für die Zukunft Österreichs (BZÖ) nicht an der Befragung zur Europawahl 2009 teil. Die Forschungsarbeit für diesen Teil des Artikels wurde im Rahmen der Austrian National Election Study (AUTNES), eines Nationalen Forschungsnetzwerks (NFN) des Fonds zur Förderung der Wissenschaftlichen Forschung (FWF) (S10905-G11), durchgeführt. 
(2) Vor dem Hintergrund der bisherigen, weithin deskriptiven Einzelanalysen zu den nationalen Wahlkämpfen in Deutschland ${ }^{44}$ und Österreich ${ }^{45}$ lässt sich zweitens annehmen, dass der Professionalitätsgrad der Parteikampagnen in Deutschland bei beiden Wahlen höher als der der österreichischen Parteien ausfallen dürfte (H2).

(3) Des Weiteren kann vor dem Hintergrund des parteizentrierten Modells der Kampagnenprofessionalisierung ${ }^{46}$ und erster vorliegender empirischer Befunde ${ }^{47}$ angenommen werden, dass in beiden Ländern und bei beiden Wahlen die Kampagnenbemühungen der regierenden Volksparteien stärker professionalisiert sind als die der oppositionellen, kleineren Parteien (H3).

(4) Diese Annahme differenzierend wird erwartet, dass der Unterschied im Professionalitätsgrad der Kampagnenstrukturen von Groß- und Kleinparteien größer als der der Kampagnenstrategien ausfallen dürfte (H4). Diese Annahme gründet sich auf den exemplarischen Befund, wonach kleinere im Bundestag vertretene Parteien erfolgreich darin sind, etwaige strukturelle Defizite in der strategischen Kampagnenkommunikation in gewissem Maße zu kompensieren. ${ }^{48}$

\section{Kampagnenstrukturen und-strategien im Vergleich}

„Zum modernen Wahlkampf braucht man drei Dinge: Geld, Geld, Geld“49, so lautete das Credo eines der erfahrensten Wahlkampfmanager Deutschlands, des ehemaligen Bundesgeschäftsführers der CDU, Peter Radunski. Wenn auch sicherlich überspitzt formuliert, steht doch außer Frage, dass die finanzielle Ressourcenausstattung bei der Planung und Durchführung einer Wahlkampagne eine zentrale Rolle spielt. Allerdings ist das Budget weder eine statische noch eine exogene Größe des Kampagnenmanagements. Vielmehr ist es ein integraler und im Wahlkampf für unterschiedliche Kommunikationsmaßnahmen variabel einsetzbarer Faktor. Auch wäre es eine unzulässige Verkürzung, die strukturellen und strategischen Facetten eines Wahlkampfes auf die Höhe des Etats zu beschränken.

44 Vgl. Jürgen Falter / Andrea Römmele, Professionalisierung deutscher Wahlkämpfe, oder: Wie amerikanisch kann es werden? in: Thomas Berg (Hrsg.), Moderner Wahlkampf. Blick hinter die Kulisse, Opladen 2002, S. 49 - 63; Jochen W. Wagner, Deutsche Wahlwerbekampagnen made in USA? Amerikanisierung oder Modernisierung bundesrepublikanischer Wahlkampagnen, Opladen 2005; Christina Holtz-Bacha, a.a.O. (Fn. 31); Jens Tenscher, a.a.O. (Fn. 11 und 12).

45 Vgl. Fritz Plasser, „Amerikanisierung“ des politischen Wettbewerbs in Österreich, in: Anton Pelinka / Fritz Plasser / Wolfgang Meixner (Hrsg.), Die Zukunft der österreichischen Demokratie, Wien 2000, S. 203 - 230; Thomas Rachbauer, Professionalisierung und Amerikanisierung im Wahlkampfmanagement der politischen Parteien in Österreich bei Nationalratswahlen in der Zweiten Republik, Dissertation an der Universität Innsbruck 2005; Andreas Lederer, Politische Werbung in der Wahlkampfarena, in: Fritz Plasser (Hrsg.), Politik in der Medienarena. Praxis politischer Kommunikation in Österreich, Wien 2010, S. $241-272$.

46 Vgl. Rachel K. Gibson / Andrea Römmele, a.a.O. (Fn. 28).

47 Vgl. Jens Tenscher, a.a.O. (Fn. 11); Rachel K. Gibson / Andrea Römmele, a.a.O. (Fn. 29); Jesper Strömbäck, a.a.O. (Fn. 21).

48 Vgl. Jens Tenscher, a.a.O. (Fn. 11 und 38).

49 Peter Radunski, Wahlkampf im Wandel. Politikvermittlung gestern - heute - morgen, in: Ulrich Sarcinelli / Jens Tenscher (Hrsg.), Machtdarstellung und Darstellungsmacht. Beiträge zu Theorie und Praxis moderner Politikvermittlung, Baden-Baden 2003, S. 183 - 198, S. 185. 
Tatsächlich gibt es eine Fülle an Maßnahmen, die diesen nur in geringem Maße belasten und dennoch von zentraler Bedeutung sind, die also dem Anspruch einer professionellen, das heißt effizient geführten Kampagne in höchstem Maße entsprechen. So entfachte zum Beispiel die FDP im EP-Wahlkampf 2004 die größte Resonanz in der Medienberichterstattung, obwohl sie über das kleinste Budget aller im Bundestag vertretenen Parteien verfügte. ${ }^{50}$

Vor dem Hintergrund der herausgehobenen Bedeutung des Etats ${ }^{51}$ soll zunächst ein Blick auf die von den Parteien veranschlagten Ausgaben in den zurückliegenden nationalen und europäischen Parlamentswahlkämpfen geworfen werden (vgl. Tabelle 1). Dabei wird

\begin{tabular}{|c|c|c|c|c|c|c|c|c|}
\hline \multicolumn{9}{|c|}{$\begin{array}{l}\text { Tabelle 1: Wablkampfausgaben der in den jeweiligen Parlamenten vertretenen Parteien } 2008 \\
\text { und } 2009\end{array}$} \\
\hline \multicolumn{2}{|c|}{ Deutschland } & SPD & $\mathrm{CDU}$ & CSU & Grüne & FDP & Linke & Gesamt \\
\hline \multirow{3}{*}{$\begin{array}{l}\text { BTW } \\
2009\end{array}$} & absolut (in Millionen Euro) & 29 & 20 & 6,5 & 4 & 5,5 & 5,0 & 70,0 \\
\hline & Cent pro Wahlberechtigtem ${ }^{\mathrm{a}}$ & 47 & 32 & 11 & 6 & 9 & 8 & 113 \\
\hline & $\varnothing$ Euro pro Wahlstimme $\mathrm{b}^{\mathrm{b}}$ & 2,9 & 1,7 & 2,3 & 0,9 & 0,9 & 1,0 & 1,6 \\
\hline \multirow{3}{*}{$\begin{array}{l}\text { EW } \\
2009\end{array}$} & absolut (in Millionen Euro) & 11 & 10 & 2,5 & 1 & 1,55 & 3,5 & 29,55 \\
\hline & Cent pro Wahlberechtigtem & 18 & 16 & 4 & 2 & 3 & 6 & 48 \\
\hline & $\varnothing$ Euro pro Wahlstimme & 2,0 & 1,2 & 1,3 & 0,3 & 0,5 & 1,8 & 1,1 \\
\hline \multicolumn{2}{|c|}{ Österreich } & SPÖ & ÖVP & FPÖ & Grüne & BZÖ & $\begin{array}{c}\text { Liste } \\
\text { Martin }\end{array}$ & Gesamt \\
\hline \multirow{3}{*}{$\begin{array}{l}\text { NRW } \\
2008\end{array}$} & absolut (in Millionen Euro) & 7 & 9 & 3 & 3 & 4 & - & 26,0 \\
\hline & Cent pro Wahlberechtigtem & 111 & 142 & 47 & 47 & 63 & - & 410 \\
\hline & $\varnothing$ Euro pro Wahlstimme & 4,9 & 7,9 & 3,5 & 5,9 & 7,7 & - & 5,7 \\
\hline \multirow{3}{*}{$\begin{array}{l}\mathrm{EW} \\
2009\end{array}$} & absolut (in Millionen Euro) & 4 & 4 & 1 & 1 & 1 & 2,25 & 13,25 \\
\hline & Cent pro Wahlberechtigtem & 63 & 63 & 16 & 16 & 16 & 35 & 209 \\
\hline & $\varnothing$ Euro pro Wahlstimme & 5,9 & 4,7 & 2,8 & 3,5 & 7,6 & 4,5 & 4,6 \\
\hline \multicolumn{9}{|c|}{$\begin{array}{l}\text { Anmerkungen: Abweichungen der für die Bundestagswahl angegebenen Ausgabenhöhen gegenüber an- } \\
\text { deren Veröffentlichungen (vgl. Helmut Rinsum / Stephanie Grill, a.a.O. (Fn. 3); Mona Krewel / Rüdiger } \\
\text { Schmitt-Beck / Ansgar Wolsing, a.a.O. (Fn. 2)) dürften dem Zeitpunkt der Datenerhebung geschuldet } \\
\text { sein. Die an dieser Stelle angegebenen Werte wurden im Abstand von einem halben Jahr zur Bundestags- } \\
\text { wahl verifiziert. Die CSU-Verantwortlichen äußerten sich weder in der Befragung noch öffentlich zu } \\
\text { ihren Wahlkampfausgaben im Jahr 2009. Der angegebene Wert basiert auf einer Schätzung (vgl. Jürgen } \\
\text { Wilke / Christian Schäfer / Melanie Leidecker, Mit kleinen Schritten aus dem Schatten. Haupt- und Ne- } \\
\text { benwahlkämpfe in Tageszeitungen am Beispiel der Bundestags- und Europawahlen 1979-2009, in: Jens } \\
\text { Tenscher, a.a.O. (Fn. 10), S. 155-179, S. 174). } \\
\text { a Eigene Berechnungen auf Basis der amtlichen Wahlstatistiken. } \\
\text { b Anzahl der erhaltenen Zweitstimmen beziehungsweise Anzahl gültiger Stimmen. } \\
\text { c Die Liste Martin trat zur Nationalratswahl } 2008 \text { nicht an. } \\
\text { Quelle: Eigene Darstellung auf Basis der Kampagnenstudien 2008-2009. }\end{array}$} \\
\hline
\end{tabular}

50 Vgl. Jens Tenscher, a.a.O. (Fn. 11), S. 73 - 75.

51 Vgl. auch Rachel K. Gibson / Andrea Römmele, a.a.O. (Fn. 29), S. 280; anders bei Lars Nord, Still the Middle Way. A Study of Political Communication Practices in Swedish Election Campaigns, in: The Harvard International Journal of Press/Politics, 11. Jg. (2006), H. 1, S. $64-76$. 
deutlich, dass sowohl deutsche als auch österreichische Parteien in die nationale Hauptwahl mehr als doppelt so viel Geld wie in die europäische Nebenwahl investierten. Dies deckt sich mit früheren Befunden zu Deutschland. ${ }^{52}$ Entsprechend kann die populäre Annahme, Parteien würden Europawahlkämpfe nur „halbherzig“ angehen, zumindest, was ihr finanzielles Engagement angeht, als gesichert gelten.

Beim Vergleich der Wahlkampfausgaben zwischen den beiden Ländern fällt zudem auf, dass in die zurückliegenden beiden Wahlkämpfe in Österreich jeweils rund vier Mal so viel Geld pro Wachberechtigtem investiert wurde wie in Deutschland. Zurückzuführen ist dies einerseits auf die herausgehobene Bedeutung der kostenintensiven Aktivitäten in Österreich, wo Plakaten und Zeitungsannoncen (,paid media“) eine zentrale Rolle zugeschrieben wird. ${ }^{53}$ Andererseits bieten die kleinen im Nationalrat beziehungsweise im EP vertretenen Parteien im Verhältnis zu den Großparteien SPÖ und ÖVP relativ viele finanzielle Ressourcen auf. Dies spricht für einen gewissen "Grundbetrag“, der für Wahlkämpfe, egal ob in Deutschland oder dem Nachbarland, mindestens ausgegeben werden muss. Bei EP-Wahlen kann dieser auf rund eine Millionen Euro, bei nationalen Parlamentswahlen auf drei bis vier Millionen Euro taxiert werden. Diese Summe muss anscheinend in einen Wahlkampf gesteckt werden, damit dieser überhaupt „ins Rollen“ kommen und Wirkungen entfalten kann. Ungeachtet der Größe des Landes und der Bevölkerungszahl bewegen sich auch deutsche Parteien nicht weit von diesem unteren Limit entfernt. Bemerkenswert ist außerdem, dass in beiden Ländern und bei beiden Wahltypen die Grünen am wenigsten Geld für den Wahlkampf bereithalten. Hierin könnte sich die andernorts aufgestellte Vermutung, „linke“ Parteien zeigten sich gegenüber kostenintensiven Kampagneninnovationen zurückhaltender als „rechte" beziehungsweise konservative Parteien, bewahrheiten. ${ }^{54}$ Allerdings deuten die Spitzenwerte für die SPD auf das Gegenteil, was für eine Relativierung der Annahme spricht.

Über die Einzelkomponente des Budgets hinaus stellt sich die Frage, welche organisatorischen Voraussetzungen die Parteien insgesamt schufen, um erfolgreich die Wahlkämpfe zu bestreiten. Eine Antwort hierauf liefert Abbildung 1, die zwischen der Professionalität der Kampagnenstrukturen im Vorfeld der europäischen Nebenwahl einerseits und der nationalen Hauptwahl andererseits differenziert. Aus Gründen der Übersichtlichkeit sind hier jeweils die Daten der beiden großen Regierungsparteien, SPD und CDU beziehungsweise SPÖ und ÖVP, zusammen dargestellt. Gleiches gilt für die „kleinen“ in den jeweiligen Parlamenten vertretenen (Oppositions-)Parteien. 55

52 Vgl. Jens Tenscher, a.a.O. (Fn. 11), S. 73.

53 Vgl. Andreas Lederer, a.a.O. (Fn. 45).

54 Vgl. Rachel K. Gibson / Andrea Römmele, a.a.O. (Fn. 28), S. 37.

55 Obwohl Regierungspartei, wird die CSU aufgrund ihrer regionalen Sonderrolle und ihrer vergleichsweise geringen Stimmenanteile der Gruppe der „kleinen“ Parteien zugerechnet. Gleiches gilt für die FPÖ, die bei der vergangenen Nationalratswahl mit 17,5 Prozent weniger als zehn Prozentpunkte hinter der zweitstärksten Kraft im Lande, der ÖVP, landete. Diese Zuordnungen sind an dieser Stelle unbedenklich, da nicht die inhaltlichen Ausrichtungen der Parteien, sondern allgemeine Strukturen und Strategien im Verhältnis zur Parteiengröße untersucht werden. 


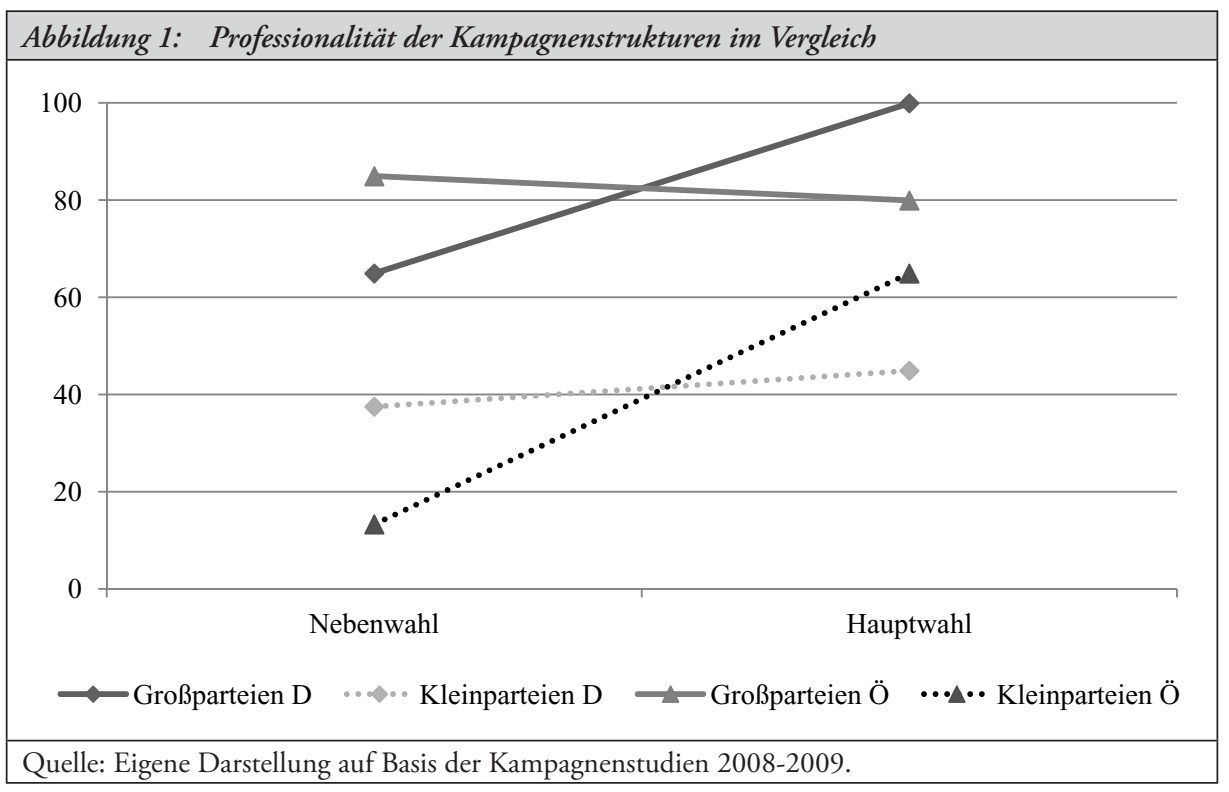

Beim Blick auf die Kampagnenstrukturen bestätigt sich in drei von vier Fällen die erste Untersuchungsannahme: Die Professionalität der organisatorischen Voraussetzungen für eine erfolgreiche Kampagne ist - mit Ausnahme der österreichischen Regierungsparteien tatsächlich bei der nationalen Hauptwahl größer als bei der europäischen Nebenwahl. Besonders deutlich fällt der Unterschied für die österreichischen Kleinparteien aus, die im Europawahlkampf 2009 mit Abstand strukturell am schlechtesten aufgestellt waren (13,3 Prozent), im Nationalratswahlkampf 2008 jedoch nur leicht unter dem Niveau von SPÖ und ÖVP blieben (64,9 vs. 79,9 Prozent).

Die zweite Untersuchungsannahme findet nur in der Hälfte der Vergleichspunkte ihre Bestätigung: Während die deutschen Großparteien sich zur Hauptwahlzeit maximal „aufrüsteten" und deutlich vor den österreichischen Pendants liegen, gilt dies für die Kleinparteien zur Europawahlkampfzeit. Insgesamt kann jedoch nicht, wie allgemein vermutet ${ }^{56}$, behauptet werden, dass deutsche Parteien per se professioneller als österreichische in Haupt- oder Nebenwahlkämpfe starten würden. Letztlich findet aber die dritte Annahme eine klare Bestätigung: In beiden Ländern und zu beiden Wahlkämpfen ist der organisatorische Professionalitätsgrad der Großparteien stärker ausgebildet als der der Kleinparteien.

Wird die Perspektive von den Kampagnenstrukturen zu den -strategien gelenkt, fällt zunächst einmal der große Abstand auf, den SPD und CDU nicht nur vor den deutschen Kleinparteien, sondern vor allen österreichischen Parteien in beiden Wahlkämpfen behaupten (vgl. Abbildung 2). Sozial- und Christdemokraten konnten sich nicht nur zur EP-, sondern auch zur Bundestagswahl strategisch bestens aufstellen. Dies mag nicht zuletzt darauf zurückzuführen sein, dass sich die deutschen Parteien im „Superwahljahr 2009“ um 
Kampagnen „aus einem Guss“ bemühten; der Europawahlkampf war in jenem Jahr ein "Vor- beziehungsweise Nebenprodukt" des Bundestagswahlkampfes. ${ }^{57}$

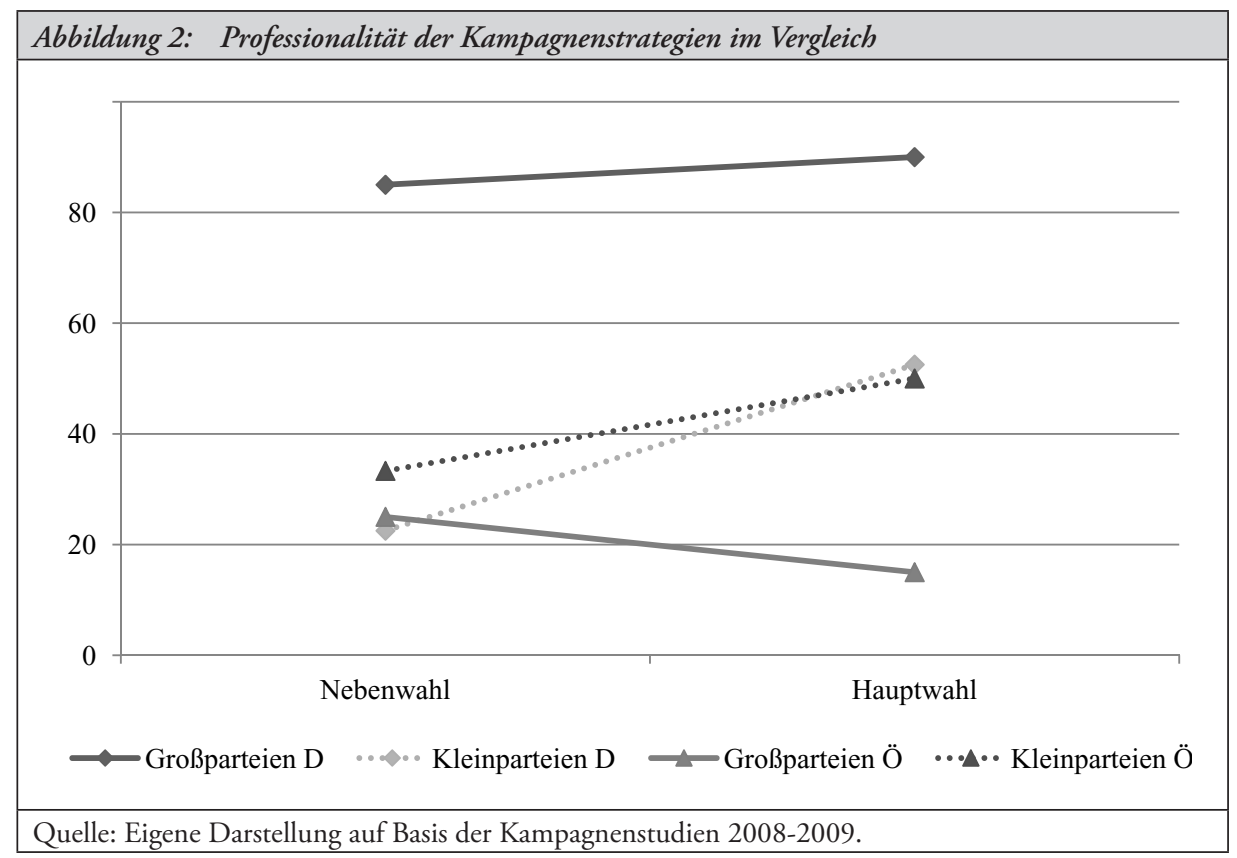

Mit Ausnahme von SPÖ und ÖVP bestätigt sich wiederum die erste Annahme, wonach der (strategische) Professionalitätsgrad im Vorfeld der Hauptwahl höher als im Europawahlkampf ausfällt. Die beiden österreichischen Regierungsparteien bewegen sich jedoch nicht nur wie die Kleinparteien in beiden Ländern im EP-Wahlkampf am unteren Ende der strategischen Professionalität (25 Prozent), sondern „verschliefen“ nahezu die vorgezogenen Neuwahlen im September 2008: Hier lagen sie in strategischer Hinsicht klar hinter den Kleinparteien, die offenkundig, der vierten Untersuchungsannahme folgend, ihr strukturelles Defizit zu kompensieren wussten. ${ }^{58}$ Den deutschen Kleinparteien gelang dies ebenfalls, allerdings in geringerem Maße.

Der strategische „Ausfall“ von SPÖ und ÖVP mag letztlich auch erklären, warum beide bei der Nationalratswahl 2008 historische Tiefstwerte erzielten: Mit nur 29,3 (SPÖ) beziehungsweise 26,0 Prozent (ÖVP) konnten sie so wenige Wähler wie niemals zuvor für sich mobilisieren. ${ }^{59}$ Die dritte Untersuchungsannahme - Großparteien agieren professioneller

57 Vgl. Sandra Brunsbach / Stefanie John / Annika Werner, Germany, in: Wojciech Gagatek, a.a.O. (Fn. 14), S. 89 - 93; Jens Tenscher, a.a.O. (Fn. 38).

58 Vgl. Herbert Kickl, Unterwegs in Richtung 20 Prozent, in: Thomas Hofer / Barbara Tóth (Hrsg.), Wahl 2008. Strategien - Sieger - Sensationen, Wien / Graz / Klagenfurt 2008, S. 55 - 64; Stefan Petzner, Reduzierung auf das Wesentliche, in: ebenda, S. $65-72$.

59 Vgl. Wolfgang C. Müller, The Snap Election in Austria, September 2008, in: Electoral Studies, 28. Jg. (2009), H. 3, S. $514-517$, S. 516. 
als Kleinparteien - trifft insofern nur für die deutschen Parteien zu. Die zweite Untersuchungsannahme findet dagegen eine klare Bestätigung: Deutsche Groß- und Kleinparteien stellten sich zur letzten Bundestagswahl strategisch professioneller auf als ihre österreichischen Pendants ein Jahr zuvor. Dabei ist der Abstand zwischen den Regierungsparteien deutlich größer als der zwischen den Kleinparteien. Den Europawahlkampf bestritten die österreichischen Kleinparteien, was die Strategien betrifft, sogar etwas professioneller als die deutschen.

Was ergibt sich aus diesen Einzelbefunden für die Frage der Gesamtprofessionalität der Kampagnenführung? Werden die beiden Komponenten - Kampagnenstrukturen und Kampagnenstrategien - abschließend zusammengeführt (vgl. Abbildung 3), zeigt sich für Deutschland ein nahezu idealtypischer Verlauf: Die Gesamtprofessionalität, mit der SPD und CDU die beiden national ausgetragenen Wahlkämpfe im Jahr 2009 bestritten, liegt deutlich über der von CSU, FDP, Grünen und Linkspartei. Auch die Entwicklung entspricht der Grundannahme und der finanziellen Ressourcenausstattung: Der Bundestagswahlkampf erforderte als Hauptwahlkampf offenkundig eine höhere Professionalität als der vorgeschaltete Europawahlkampf. Insoweit bestätigen sich frühere Befunde. ${ }^{60}$

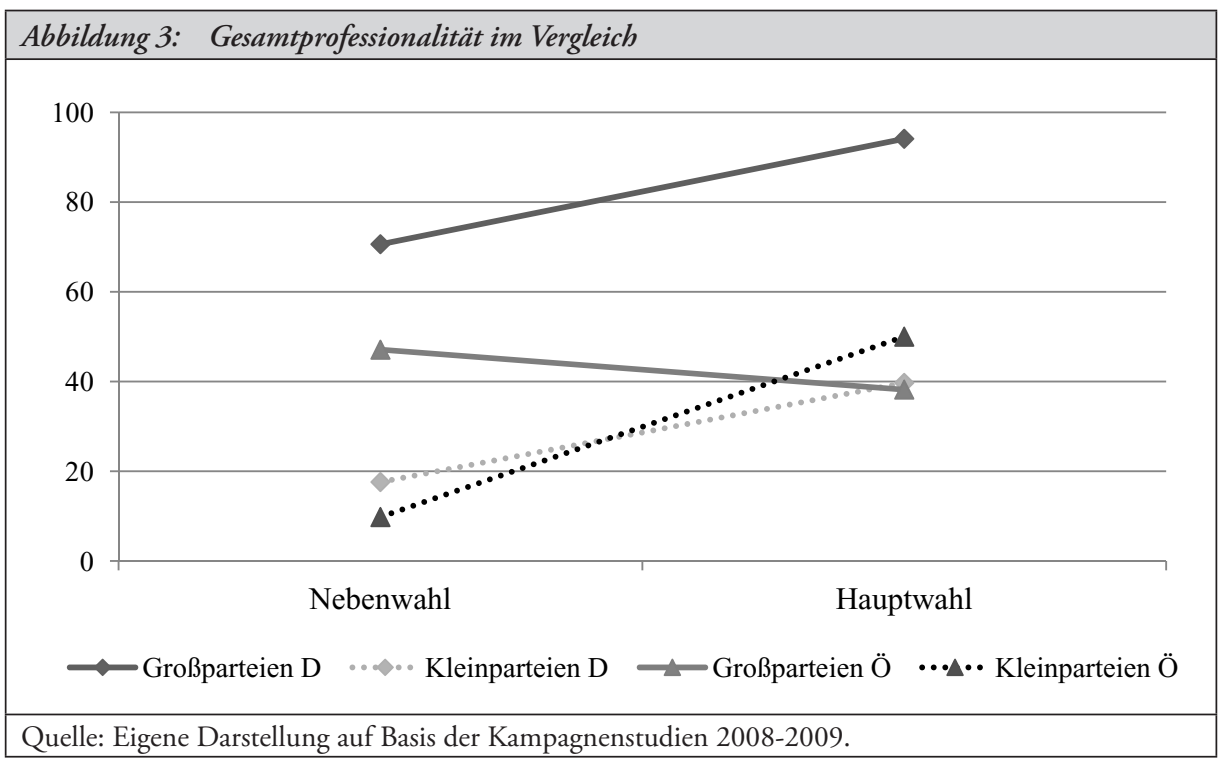

Überraschenderweise ist dies jedoch im österreichischen Kontext anders: Hier gelang es den Kleinparteien nicht nur, sich zur nationalen Hauptwahl professioneller als ihre deutschen Pendants aufzustellen, sondern auch ihre österreichischen Kontrahenten, SPÖ und ÖVP, zu übertrumpfen. Dies konnte jedoch nur gelingen, weil die beiden Regierungsparteien die vorgezogene Nationalratswahl 2008 hinsichtlich des Aufbaus professioneller Strukturen und des Einsatzes entsprechender Strategien „verpassten“ - und sogar unter dem Niveau 
der folgenden EP-Wahl blieben. Inwieweit sich hierin ein generelles Muster zeigt oder sich der - für Österreich nicht ungewöhnliche - Umstand vorgezogener Neuwahlen manifestiert, gilt es weiter zu überprüfen.

\section{Diskussion der Befunde und Ausblick}

Alles in allem unterstreichen die quantitativen Befunde nachdrücklich, dass nicht nur die Massenmedien und Bürger zwischen Haupt- und Nebenwahlen differenzieren, sondern dass dies auch für die politischen Protagonisten zutrifft. Für den deutschen Kontext hat sich der Vorrang des Bundestagswahlkampfes nachhaltig und für beide Parteiengruppen bestätigt. ${ }^{61}$ In Österreich konnten demgegenüber lediglich die kleinen im Parlament vertretenen Parteien, wie erwartet, im Vergleich von EP- und Nationalratswahlkampf, was die Professionalität ihrer Kampagnenstrukturen und -strategien angeht „zulegen“. SPÖ und ÖVP gelang dies nicht; sie gingen - auf niedrigem Niveau - professioneller in die Europawahl als in die vorgezogene Nationalratswahl 2008. Dies mag auch ein Ergebnis des Austauschs der Wahlkampfverantwortlichen nach der für beide Parteien desaströs verlaufenen nationalen Parlamentswahl gewesen sein. Dessen ungeachtet bleibt festzuhalten, dass sich deutsche und österreichische Kleinparteien aber, entgegen der ursprünglichen Annahme, auf etwa dem gleichen Professionalitätsniveau bewegen; bei den österreichischen Großparteien zeichnet sich demgegenüber ein deutlicher Nachholbedarf ab. ${ }^{62}$

In methodischer Hinsicht bleibt anzumerken, dass die vorliegende Studie mit dem Versuch, die Professionalität politischer Kommunikationsmaßnahmen zu quantifizieren und damit vergleichbar zu machen, ein noch weithin unbeackertes Feld betritt. ${ }^{63}$ Dabei basiert der Vergleich auf einer Auswahl an Indikatoren, die genauso wie eine etwaige Gewichtung der Variablen hinterfragt werden müssen. Die Annahme, dass mit diesen Komponenten der Raum des Kampagnenmanagements adäquat erfasst werden kann, wird zwar durch die Antworten der österreichischen Wahlkampfmanager auf eine entsprechende Nachfrage in den Interviews bestätigt. Ob die Indikatoren auch raum- und zeitungebunden valide sind, kann jedoch nur im Rahmen weiterer, zeit- und ländervergleichender Studien überprüft werden. Insofern sollte hier vor allem ein Auftakt für weitere komparativ angelegte Kampagnenstudien gemacht werden, in denen auch das methodische Design auf den Prüfstand gestellt wird. Neben der Validität stellt sich schließlich die Frage der Reliabilität der Befunde, die durch die (begründete) Berücksichtigung subjektiver Eindrücke der Wahlkampfverantwortlichen nolens volens gemindert wird. Auch dafür sind zukünftig objektiv und subjektiv gewonnene Indikatorensets zu überprüfen.

Ziel weiterer Kampagnenstudien muss es letztlich sein, nicht nur nach der Effizienz der gewählten Maßnahmen, sondern nach deren „Erfolg“ zu fragen. Entsprechende Evaluationsstudien müssen in Abhängigkeit zu den gewählten Zielen und unter Berücksichtigung von Kampagneninhalten stehen. Diese variieren jedoch erheblich. So kann der „Erfolg“ zum Beispiel in der Steigerung von Medienresonanz, in der Maximierung von Wählerstim-

61 Vgl. Jens Tenscher, a.a.O. (Fn. 11 und 38).

62 Vgl. ders., „Die neue Wahl“. Ein SPÖ-Wahlkampf aus dem Stand, in: Thomas Hofer / Barbara Tóth, a.a.O. (Fn. 58), S. $32-45$.

63 Vgl. Rachel K. Gibson / Andrea Römmele, a.a.O. (Fn. 29); Jesper Strömbäck, a.a.O. (Fn. 21). 
men, in positiven Urteilen oder in finanzieller Unterstützung zum Ausdruck kommen. Diese Dimensionen zu quantifizieren und sie kausal auf bestimmte Kampagnenstrukturen oder -strategien zurückzuführen, dürfte dem Versuch gleichen, einen Pudding an die Wand zu nageln. Dessen unbenommen muss es im Interesse der politischen Kommunikationsund Kampagnenforschung liegen, diesen Weg weiter zu verfolgen. Nur so kann eine Annäherung an die Art und Weise der Wahlkampfführung, deren Ursachen und Konsequenzen gelingen.

\section{Anhang: Professionalisierungsindex}

Der Professionalisierungsindex setzt sich aus zwei jeweils additiven Teilindizes zusammen, anhand derer die Professionalität (a) der Kampagnenstrukturen und (b) der Kampagnenstrategien der einzelnen Parteien gemessen wird. Beide Teilindizes umfassen mehrere Indikatoren, deren Vorhandensein beziehungsweise deren Ausmaß jeweils anhand von Punkten festgehalten wird. Die Punktevergabe für die einzelnen Indikatoren erfolgt in der Regel anhand objektiver Kriterien und ergänzend auf Basis der subjektiven Einschätzungen der jeweils für eine Wahlkampagne verantwortlichen Parteienvertreter (markiert mit *).

\section{Indikatoren Kommunikationsstrukturen (8 Indikatoren, 24 Punkte maximal)}

- Größe der Wahlkampfbudgets

Die Punkteverteilung richtet sich nach der Höhe der Wahlkampfausgaben einer Partei pro Wahlberechtigtem. Dabei gilt: 0 bis 10 Cent $=1$ Punkt; 11 bis 20 Cent $=2$ Punkte; 21 bis 30 Cent $=3$ Punkte; 31 bis 40 Cent $=4$ Punkte; mehr als 40 Cent $=5$ Punkte.

- Größe des Mitarbeiterstabs

Die Punkteverteilung richtet sich nach der Anzahl der auf nationaler Ebene in die Planung, Organisation und Durchführung der Wahlkampagne involvierten institutionalisierten (das heißt in der Partei arbeitenden), dauerhaften und temporär eingestellten Mitarbeiter. Dabei gilt: 0 bis 10 Mitarbeiter $=0$ Punkte; 11 bis 30 Mitarbeiter $=1$ Punkt; 31 bis 50 Mitarbeiter = 2 Punkte; mehr als 50 Mitarbeiter = 3 Punkte.

- Ausmaß der Zentralisierung der Kampagnenorganisation*

Die Punkteverteilung richtet sich nach dem Ausmaß der auf nationaler Ebene in der Hände einer Leitungsstruktur gebündelten Zentralisierung des Kampagnenmanagements. Dabei bezieht sich die Zentralisierung auf die organisatorische, strategische und inhaltliche Ausrichtung der Parteienkampagne. Es gilt: dezentralisiert = 0 Punkte; teilweise zentralisiert = 1 Punkt; vollständig zentralisiert = 2 Punkte.

- Ausmaß der Externalisierung

Die Punkteverteilung richtet sich nach der Anzahl der für allgemeine und/oder spezifische Wahlkampftätigkeiten, wie zum Beispiel Werbung, News- und Ereignismanagement, Mediaplanung, Meinungsforschung, Zielgruppenansprache, Internetkampagne etc., zeitlich befristet engagierten Agenturen und entsprechender Politikvermittlungsexperten beziehungsweise Berater. Dabei gilt: keine Agentur/Berater = 0 Punkte; 1 bis 2 Agenturen/Berater = 1 Punkt; 3 bis 4 Agenturen/Berater = 2 Punkte; mehr als 4 Agenturen/Berater $=3$ Punkte. 
- Ausdifferenzierung der internen Kommunikationsstrukturen

Die Punkteverteilung richtet sich nach Vorhandensein und Nutzung „neuer“ Kommunikationsmedien zur wahlkampfspezifischen internen Kommunikation und Mitgliedermobilisierung. Hierzu wird für jede der folgenden drei Kommunikationskanäle festgehalten, ob entsprechende Strukturen genutzt (1 Punkt) oder nicht genutzt (0 Punkte) wurden: (a) Intranet, (b) Direct Mailings sowie Telemarketing, das heißt (c) Telefon, (d) E-Mail und (e) SMS/MMS. Maximal können fünf Punkte erreicht werden.

- Art und Umfang der Rückkopplung

Die Punkteverteilung richtet sich nach der Nutzung zweier zentraler Methoden, geplante Kampagnenmaßnahmen zu testen sowie zielgruppenspezifische und repräsentative Meinungen zu erfassen. Hierzu wird festgehalten, ob (a) Meinungsumfragen in Auftrag gegeben beziehungsweise eigenständig durchgeführt wurden und (b) Fokus-Gruppen getestet wurden. Für beide Rückkopplungswege wird jeweils festgehalten, ob sie genutzt (1 Punkt) oder nicht genutzt (0 Punkte) wurden. Maximal können zwei Punkte erreicht werden.

- Ausmaß der Gegnerbeobachtung

Hier wird festgehalten, ob eigenständige Strukturen zur Beobachtung der politischen Kontrahenten aufgebaut wurden. Sollte dies geschehen sein, wird 1 Punkt vergeben, ansonsten 0 Punkte.

- Kampagnendauer

Hier wird die Gesamtdauer der Vorbereitungen, Planungen und Durchführung einer Kampagne bis zum Wahltag festgehalten. Dabei gilt: 0 bis 5 Monate $=0$ Punkte; 6 bis 12 Monate = 1 Punkt; 1 bis 2 Jahre = 2 Punkte; mehr als 2 Jahre = 3 Punkte.

\section{Kommunikationsstrategien (7 Indikatoren, 26 Punkte maximal)}

- Ausmaß der Zielgruppenorientierung

Die Punkteverteilung richtet sich nach der Anzahl der seitens der Parteien identifizierten Zielgruppen, die spezifisch erreicht und mobilisiert werden sollen. Dabei gilt: keine spezielle Zielgruppe = 0 Punkte; 1 bis 2 spezielle Zielgruppen $=1$ Punkt; 3 bis 4 spezielle Zielgruppen = 2 Punkte; 5 und mehr spezielle Zielgruppen = 3 Punkte.

- Ausmaß der Narrowcasting-Aktivitäten

Die Punkteverteilung richtet sich danach, wie viele Maßnahmen der zielgruppenspezifischen Direktkommunikation zum Einsatz kamen. Dabei wird für jede der folgenden vier Maßnahmen festgehalten, ob sie im Wahlkampf genutzt wurde (1 Punkt) oder nicht (0 Punkte): (a) Direct Mailings, Telemarketing, das heißt (b) Telefon, (c) E-Mail und (d) SMS/MMS, (e) Canvassing/Hausbesuche, (f) Informationsstände, Feste, Sonstiges. Maximal können sechs Punkte erreicht werden.

- Relevanz der paid media*

Hier wird die subjektive Einschätzung der Wahlkampfverantwortlichen hinsichtlich des Stellenwerts, den sie verschiedenen Werbemedien zumaßen, festgehalten. Im Fragebogen wurde hierbei mit einer 5er Skala ( 1 = „völlig unwichtig“ bis 5 = „sehr wichtig“) operiert. Punkte werden nur für diejenigen bezahlten Werbekanäle vergeben, die als 
„wichtig“ oder „sehr wichtig“ (Wert > 4), eingestuft wurden. Dabei wurde zwischen folgenden fünf Werbemedien unterschieden: (a) Parteienwerbung im Fernsehen, (b) Parteienwerbung im Kino, (c) Parteienwerbung im Radio, (d) Plakatwerbung, (e) Anzeigen in Zeitschriften und Zeitungen. Maximal können fünf Punkte erreicht werden.

- Relevanz der free media*

Hier wird die subjektive Einschätzung der Wahlkampfverantwortlichen hinsichtlich des Stellenwerts der Präsenz ihrer Partei beziehungsweise ihres Spitzenkandidaten in verschiedenen massenmedialen Formaten festgehalten. Im Fragebogen wurde hierbei mit einer 5er Skala ( 1 = „völlig unwichtig“ bis 5 = „sehr wichtig“) operiert. Punkte werden nur für diejenigen „kostenfreien“ Formate vergeben, die als „wichtig“ oder „sehr wichtig“ (Wert > 4), eingestuft wurden. Dabei wurde zwischen folgenden sechs Formaten beziehungsweise Plattformen unterschieden: (a) überregionale Tages-/Wochenzeitungen, politische Zeitschriften, (b) regionale/lokale Printmedien, (c) Boulevard-Printmedien (inklusive Bild-Zeitung), (d) Radio, (e) Internet (Website, Weblogs, soziale Netzwerke, Videoportale), (f) Fernsehen. Maximal können sechs Punkte erreicht werden.

- Relevanz der Talkshowisierung*

Hier wird die subjektive Einschätzung der Wahlkampfverantwortlichen hinsichtlich des Stellenwerts der Präsenz ihres Spitzenkandidaten (a) in politischen Talkshows und (b) in Prominenten- und sonstigen unpolitischen Talkshows festgehalten. Im Fragebogen wurde hierbei mit einer 5er Skala (1 = „völlig unwichtig“ bis 5 = „sehr wichtig“) operiert. Punkte werden nur vergeben, wenn ein Format als „wichtig“ oder „sehr wichtig“ (Wert > 4), eingestuft wurde. Maximal können zwei Punkte erreicht werden.

- Ausmaß des Ereignis- und Newsmanagements*

Hier wird die subjektive Einschätzung der Wahlkampfverantwortlichen hinsichtlich des Ausmaßes an inszenierten Ereignissen im Rahmen der Parteienkampagne festgehalten. Dabei gilt: keine Inszenierung spezifischer Medienereignisse = 0 Punkte; vereinzelte Inszenierungen spezieller Medienereignisse $=1$ Punkt; häufige Inszenierungen spezieller Medienereignisse $=2$ Punkte.

- Ausmaß der Personalisierung*

Hier wird die subjektive Einschätzung der Wahlkampfverantwortlichen hinsichtlich der Bedeutung des Spitzenkandidaten im Rahmen der Parteienkampagne im Verhältnis zur Gewichtung von Issues festgehalten. Dabei gilt: vorwiegend themenorientierte Kampagne $=0$ Punkte; themen- und kandidatenorientierte Kampagne = 1 Punkt; vorwiegend kandidatenorientierte Kampagne = 2 Punkte. 